\title{
Author Index to Volume 33
}

Abdulla R, 43

Abeling NGGM, 412

Abrams SA, 92

Abran D, 336

Acra SA, 215

Adams RM, 313

Adderson EE, 307

Ailabouni AH, 568

Akamatsu $\mathrm{H}, 159$

Akino T, 209

Akusjärvi G, 5

Albano F, 205

Alessio M, 205

Amendt B, 129

Amiel SA, 497

Anderson WF, 313

Ando M, 302

Aperia A, 5

Aprille JR, 433

Arens M, 36

Armstead WM, 164, 609

Arvilommi H, 548

Arvola T, 548

Aszterbaum M, 418

Atkinson SA, 118

Austgulen R, 82

Avison MJ, 595

Baan J, 295

Baker B, 136

Bakker HD, 412

Balaraman V, 365

Baley PA, 313

Bao W, 323

Barnard JA, 215

Barrington K, 190

Battaglia FC, 590

Beaufrère $B, 101$

Bell EF, 106

Belsha CW, 136

Bender KS, 289

Benelli C, 284

Berenson GS, 323

Bergstrom W, 92

Berkowitz ID, 48

Bertolo RFP, 118

Bhatia J, 487

Bier DM, 106

Birdwell A, 36

Blizzard RM, 278

Bolaños JP, 66

Bonne G, 284

Bonuccelli CM, 519

Bossi G, 506

Boulware SD, 497

Boydstun II, 595

Braga S, 201

Brandt M, 313

Bremner WJ, 514

Brenner RE, 390

Brewer ED, 136

Brown GC, 603

Brown RK, 247

Bucher H-U, 56

Busija DW, 164

Cai Z, 225

Caprio S, 497

Carey D, 313

Carlsson B, 475

Carnielli VP, 583

Carroll WL, 307
Carver TD, 493

Celsi G, 1, 5

Chan W, 190

Chapleau MW, 649

Chapman TE, 583

Chaussain M, 284

Chemtob S, 336

Chen Y, 171

Chessex P, 347

Cinti B, 506

Clancy RL, 554

Clark HF, 394

Clot J-P, 284

Connelly A, 77

Connuck DM, 628

Contant CF, 598

Cope M, 603

Cotton RGH, 125

Covert RF, 373

Cripps AW, 554

Crissinger KD, 543

Cross JH, 77

Curatola G, 506

Dalinghaus M, 267

Darlington $\mathrm{G}, 313$

David-Cu R, 332

Davidson D, 242

de Bont ESJM, 380

Deckelbaum RJ, 15

Degenhart HJ, 583

de $\mathrm{Haan} \mathrm{HH}, 185$

de Haan J, 185

de Leij LHFM, 380

Delpy DT, 603

De Meirleir L, 284

de Muinck Keizer-Schrama SMPF, 577

Dennery PA, 87

Dianzani I, 125

Diry M, 284

Dooling EC, 433

Dorland B, 412

Drop SLS, 577

Drummond WH, 181

Drwinga $H, 441$

Duc G, 56

Dunkel L, 514

Dykes W, 215

Easa D, 365

Eaves LJ, 645

Edwards AD, 56, 603

Efstatopoulos T, 583

Ekre HP, 475

Ekstrand BC, 195

Ellerine NP, 445

Elsas LJ, 445

Epstein ML, 527

Ernst SG, 433

Eronen M, 615

Espevik T, 82

Ewald U, 97

Fabian J, 273

Feingold KR, 418

Fennessey PV, 590

Fernandez H, 336

Ferrero GB, 125

Ferretti G, 506

Feste AS, 564

Fetter WPF, 380

Figueras J, 112
Finegold M, 313

Fineman JR, 341

Finn $\mathrm{KC}, 365$

Fisher J, 394

Fomon SJ, 573

Fournier MA, 510

Frank L, 171, 632

Freezer NJ, 261

Frestedt J, 452

Fukao T, 429

Gadian DG, 77

Gaudio KM, 595

Gehrz R, 452

Geoffroy V, 284

Gerberding K, 19

Gerding AM, 267

Gershwin ME, 233

Gervasio CT, 533

Ghishan FK, 215

Gibson RA, 425

Gibson RL, 10

Gidday JM, 620

Glantz SA, 359

Glasscock GF, 144

Gleeson M, 554

Gluckman PD, 61

Goddard-Finegold J, 598

Goggin M, 425

Golub MS, 233

Graham TW, 233

Granot E, 15

Gratama JWC, 267

Greenwood RS, 152

Greisen G, 52

Guardamagna O, 125

Guarino A, 205

Guerra-Romero L, 510

Gunn AJ, 61

Gunning MI, 61

Gustafsson J, 97

Hählen K, 577

Hahn-Zoric M, 475

Hanson LÅ, 475

Haque AK, 487

Harper RG, 32, 466

Harris MC, 27

Hasaart THM, 185

Hashimoto T, 429

Hauhart RE, 72

Hayden WR, 48

Hay Jr WW, 493

Heinze E, 390

Heitjan DF, 405

Heitlinger LA, 568

Hendrickx AG, 233

Hewitt JK, 645

Higurashi M, 159

Hill KE, 332

Hintz RL, 136

Hoath SB, 418

Hokken-Koelega ACS, 577

Holmes G, 527

Hornickova Z, 36

Hoshi $J, 653$

Hunkeler N, 452

Hunter JV, 77

Ikegami M, 256

Ilonen J, 501

Isolauri E, 548

Ito $\Upsilon, 302$
Iwamoto HS, 639

Jaeken J, 540

Jeansson S, 475

Jeneson JAL, 412

Jenkins M, 469

Jimenez R, 112

Jobe A, 256

Jones Jr MD, 48

Jones TW, 497

Jyonouchi H, 458

Käär M-L, 501

Kabe K, 653

Kaila M, 548

Kalhan SC, 101

Kamran S, 32, 466

Kay M, 313

Keen CL, 233

Keeney SE, 177

Kelly FJ, 247

Kerrigan JR, 278

Ketelsen U-P, 201

Key Jr LL, 384

Kim JD, 144

Kirby ML, 43, 628

Kirkham FJ, 77

Klautz RJM, 295

Klein PD, 445

Kloster B, 36

Knip M, 501

Koers JH, 267

Kohl S, 469

Konduri GG, 533

Koppel R, 352

Kuipers JRG, 267

Kuroki Y, 209

Kuwahara T, 429

Lafeber HN, 101

Lanford R, 313

Langley SC, 247

Larsson A, 97

Lavoie J-C, 347

Lazarow PB, 441

Leatherbury L, 628

Ledley FD, 313

Lee PDK, 136

Leffler CW, 164, 609

Leonard JV, 77

Leroux J-P, 284

Lewis D, 313

Li BUK, 568

Liabakk N-B, 82

Liechti-Gallati S, 201

Lien E, 82

Lifschitz CH, 564

Lipp AE, 56

Lissens W, 284

Liu F, 136

Lonnerdal B, 118, 233

Lou $\mathrm{H}, 52$

Maeda M, 557

Majamaa H, 548

Makrides M, 425

Makrides V, 510

Mallard EC, 61

Marsac C, 284

Marshall T, 129

Marshiseppe I, 506

Martens A, 380

Martha Jr PM, 278 
Marti-Henneberg C, 112

Martin CG, 598

Massó J, 112

Mathews MJ, 177

Matsumoto S, 209

Mayock DE, 10

McCleery R, 487

McClung HJ, 568

McCormick DC, 603

McLaughlin GE, 632

McMenamy KR, 251

McNearney T, 36

Medina JM, 66

Meeker RB, 152

Mehendale HM, 225

Mehta R, 32, 466

Menon GK, 418

Merkel P, 497

Merrill DC, 649

Meschia G, 590

Messini L, 506

Meuzelaar KJ, 267

Michael LH, 598

Michaud GA, 221

Miettinen PJ, 481

Miller CE, 400

Mills J, 469

Milstein JM, 359

Mirro R, 164, 609

Moen R, 313

Molina J, 112

Momma K, 302

Moores Jr RR, 590

Moser AB, 441

Moser AH, 418

Moser HW, 441

Moskowitz WB, 645

Moslen MT, 487

Mosteller M, 645

Mueller W, 394

Murayama K, 152

Murayama S, 152

Murphy TD, 10

Murray RD, 568

Nagata S, 557

Nance WE, 645

Nankervis CA, 400

Näntö-Salonen K, 144

Narkewicz MR, 493

Nelson SE, 573

Nerlich A, 390

Nicolai T, 261

Nishida H, 653

Nowicki PT, 400

Nunez FL, 332

Offidani AM, 506

Ogra PL, 394

Ohtsuka Y, 557

Oishi M, 653
Okken A, 380

Olin KL, 233

Orii T, 429

Oshika E, 209

Osterhaus ADME, 475

Palmer C, 405

Parfenova H, 609

Park TS, 620

Payne NR, 452

Pearson HA, 497

Permutt S, 519

Pietsch J, 215

Pokorny W, 313

Polak MJ, 181

Polberger S, 118

Polk DB, 215

Polmar SH, 36

Ponsot G, 284

Ponzone A, 125

Ponzone R, 125

Potter A, 603

Powell DR, 136

Powers PA, 568

Pryds O, 52

Puukka R, 501

Quinn A, 307

Rabinovitch M, 352

Rabinowitz R, 15

Rantala I, 548

Rasmussen C, 433

Rassin DK, 177, 487

Ratner L, 36

Reitberg CC Th, 590

Reynolds EOR, 603

Rhead W, 129

Rider E, 256

Riepenhoff-Talty M, 394

Ries WL, 384

Risteli J, 501

Risteli L, 501

Rivera Jr A, 106

Roberton D, 475

Roberts RL, 405

Robillard JE, 649

Rodgers PA, 87

Rodriguiz RM, 384

Roettger V, 129

Rogol AD, 278

Rosenfeld RG, 144

Rossi T, 394

Rothenberg S, 313

Roy M-S, 336

Rudolph AM, 639

Ruitenbeek W, 412

Sacks AI, 215

Sakai A, 429

Sakiyama Y, 209

Salas-Salvadó J, 112
Samson G, 380

Santos CD, 242

Santos MJ, 441

Sauer PJJ, 101, 583

Schaekel K, 394

Schieken RM, 645

Schlesinger M, 15

Scholte HR, 412

Schreiber MD, 373

Segar JL, 649

Segawa M, 159

Sengers RCA, 412

Shackelford PG, 307

Shalit M, 27

Shapiro LJ, 321

Sherwin RS, 497

Shibata M, 164, 609

Shimada M, 159

Shneider BL, 221

Shoemaker L, 92

Short BL, 289

Siegel NJ, 595

Siimes MA, 514

Sim H, 365

Simmer K, 425

Simonetti O, 506

Skov L, 52

Sloan HR, 568

Slott EF, 43

Sly PD, 261

Soifer SJ, 341

Soriano HE, 313

Sosenko IRS, 327

Southwick FS, 27

Spada M, 125

Srinivasan SR, 323

Standaert TA, 10

Steendijk P, 295

Steinbrugger B, 273

Stevens EL, 365

Stevenson DK, 87, 195

Stijnen T, 577

Storch GA, 36

Suchy FJ, 221

Sulkers EJ, 101, 583

Sun B, 256

Sunehag A, 97

Sylvester JT, 519

Szczepankiewicz JR, 144

Tamborlane WV, 497

Tarallo L, 205

Täuber MG, 510

Teitel DF, 295, 639

Teller WM, 390

Theodorou AA, 533

Thompson CC, 164

Thulin G, 595

Thurston JH, 72

Tomita Y, 458

Towfighi J, 405
Traystman RJ, 48, 289

Tso P, 543

Turck D, 564

Tureen JH, 510

Uhnoo I, 394

Uriu-Hare JY, 233

Usmani SS, 32, 466

Uyehara CFT, 365

van Bel F, 295

Van Belle H, 185

van de Berg M, 267

Van Den Bogert C, 412

van Doorn JWD, 577

Van Geet C, 540

Van Gennip AH, 412

van Goudoever JB, 101, 583

van Raan J, 380

Van Reempts JLH, 185

Varma DR, 336

Veijola R, 501

Velasquez OR, 543

Veldhuis JD, 278

Vetter U, 390

Vieira N, 92

Virtanen E, 548

Vreman HJ, 87, 195

Wakazono A, 429

Walker LK, 289

Walther FJ, 332

Wanders RJA, 412

Wang Z, 118

Wang Z-M, 1, 5

Wapnir RA, 32, 466

Welch TR, 92

West AB, 221

Whitington $P, 313$

Whitney PL, 171

Williams CE, 61

Williams ML, 418

Wilson PM, 307

Wolff G, 201

Wong J, 341

Woodrum DE, 10

Woo SLC, 313

Wyatt JS, 603

Yabuta K, 557

Yamaguchi S, 429

Yamashiro Y, 557

Yamashita Y, 598

Yergey AL, 92

Yoder MC, 19

Yoon KL, 433

Zachman RD, 251

Zach MS, 273

Zhang L, 458

Zhu X-H, 595

Ziegler EE, 573

Zijlstra WG, 267

Zuckerman S, 609 